\title{
Microtubule-Associated Protein 1B Controls Directionality of Growth Cone Migration and Axonal Branching in Regeneration of Adult Dorsal Root Ganglia Neurons
}

\author{
Céline Bouquet, ${ }^{1}$ Sylvia Soares, ${ }^{1}$ Ysander von Boxberg, ${ }^{1}$ Michèle Ravaille-Veron, ${ }^{1}$ Friedrich Propst, ${ }^{2}$ and \\ Fatiha Nothias ${ }^{1}$ \\ ${ }^{1}$ Unité Mixte de Recherche 7101, Centre National de la Recherche Scientifique-Université Pierre et Marie Curie, Laboratory Neurobiologie des Signaux \\ Intercellulaires, Institut Fédératif de Recherche-Biologie Intégrative, Université Pierre et Marie Curie, 75005 Paris, France, and ${ }^{2}$ Institute of Biochemistry \\ and Molecular Cell Biology, Vienna Biocenter, University of Vienna, A-1030 Vienna, Austria
}

\begin{abstract}
During development, microtubule-associated protein 1B (MAP1B) is one of the earliest MAPs, preferentially localized in axons and growth cones, and plays a role in axonal outgrowth. Although generally downregulated in the adult, we have shown that MAP1B is constitutively highly expressed in adult dorsal root ganglia (DRGs) and associated with central sprouting and peripheral regeneration of these neurons. Mutant mice with a complete MAP1B null allele that survive until adulthood exhibit a reduced myelin sheath diameter and conductance velocity of peripheral axons and lack of the corpus callosum. Here, to determine the function of MAP1B in axonal regeneration, we used cultures of adult DRG explants and/or dissociated neurons derived from this map1b-/- mouse line. Whereas the overall length of regenerating neurites lacking MAP1B was similar to wild-type controls, our analysis revealed two main defects. First, map1b-/- neurites exhibited significantly (twofold) higher terminal and collateral branching. Second, the turning capacity of growth cones (i.e., "choice" of a proper orientation) was impaired. In addition, lack of MAP1B may affect the post-translational modification of tubulin polymers: quantitative analysis showed a reduced amount of acetylated microtubules within growth cones, whereas the distribution of tyrosinated or detyrosinated microtubules was normal. Both growth cone turning and axonal branch formation are known to involve local regulation of the microtubule network. Our results demonstrate that MAP1B plays a role in these processes during plastic changes in the adult. In particular, the data suggest MAP1B implication in the locally coordinated assembly of cytoskeletal components required for branching and straight directional axon growth.
\end{abstract}

Key words: microtubule-associated protein 1B; dorsal root ganglia; microtubules; MAP1B knock-out; axonal stability; growth cone turning; axonal spreading; outgrowth

\section{Introduction}

Outgrowth and stabilization of axons during development of the nervous system and reorganization of axonal connections in the adult are based on the dynamic rearrangement of the cytoskeleton (for review, see Baas and Luo, 2001; Dent and Gertler, 2003). Growth-promoting or directional cues in the axonal environment are sensed by the growth cone, a highly mobile structure at

\section{Received Jan. 19, 2004; accepted June 23, 2004.}

This work was supported by grants from Institut de Recherche pour la Moelle Epinière (F.N.), Fondation pour la Recherche Medicale (F.N.), Association pour la Recherche Sur la Sclérose En Plaques (F.N.), Association Française contre les Myopathies (fellowship to S.S.), the Austrian Science Fund (Fonds zur Förderung der Wissenschaftlichen Forschung; Grant F607; to F.P.), the Austrian Exchange Service (Österreichischer Austauschdienst; to F.P.), Scientific and Technological Cooperation with France, and Program Amadeus 2004-2005 (project number 15/2004; to F.P. and F.N.). We thank Dr. G. Gallo for advice on quantitative analysis of immunostaining. We are grateful to Drs. G. G. Gundersen, Itzhak Fischer, and D. J. Hartmann for providing us with anti-tyrosinated and anti-detyrosinated tubulin superglu-, MAP1B-, and laminin antibodies. We also thank M. Lambert and Dr. R. M. Mège for help with the video-microscopy (plateform for cellular imaging; Institut Fédératif de Recherche 83 Biologie Intégrative).

Correspondence should be addressed to Dr. Fatiha Nothias, Unité Mixte de Recherche 7101, Centre National de la Recherche Scientifique-Université Pierre et Marie Curie, Laboratory Neurobiologie des Signaux Intercellulaires, Université Pierre et Marie Curie, Case 2,7 Quai Saint Bernard, 75005 Paris, France. E-mail: fatiha.nothias@snv.jussieu.fr. DOI:10.1523/JNEUROSCI.2254-04.2004

Copyright $\odot 2004$ Society for Neuroscience $\quad 0270-6474 / 04 / 247204-10 \$ 15.00 / 0$ the distal end of the growing axon (for review, see GordonWeeks, 1991b). This information is then translated into a coordinated assembly of cytoskeletal proteins, in particular those constituting microtubules (MTs) (Gordon-Weeks, 1991a, 1993; Tanaka and Kirschner, 1991; Tanaka et al., 1995), and actin filaments (AFs). Axonal outgrowth is thought to require a cross talk between AFs and MTs (Gordon-Weeks, 1991c, 1993; Lin and Forscher, 1993; Challacombe et al., 1996), as has been demonstrated for growth cone turning (Challacombe et al., 1996) and axonal branching (Dent and Kalil, 2001).

The dynamic properties of MTs are regulated by diverse cellular factors, in particular by microtubule-associated proteins (MAPs) (Matus, 1990; Hirokawa, 1994). One of the earliest MAPs to be expressed during development is MAP1B (Tucker and Matus, 1988; Gordon-Weeks and Fischer, 2000). The preferential localization of MAP1B in the distal axonal portion, including the growth cone, suggested a role in axon outgrowth (Black et al., 1994; Bush et al., 1996; Mack et al., 2000). Additional evidence for this has come from the generation of several MAP1B mutant mice (Edelmann et al., 1996; Takei et al., 1997; Meixner et al., 2000), despite some discrepancies concerning the severity of the 
resulting phenotype. Thus, for example, cultured neurons from hypomorphous MAP1B mutant mice that die at birth exhibit a defect in axonal outgrowth (Gonzalez-Billault et al., 2001, 2002). For another mutant mouse line (Meixner et al., 2000), selective defects in the central and peripheral nervous systems have been observed that persist until adulthood.

In addition to its interaction with microtubules, MAP1B can also bind to AFs, and it may serve as a link between MTs and actin in the growth cone (Pedrotti and Islam, 1996; Tögel et al., 1998; Noiges et al., 2002). Furthermore, there is growing evidence that MAP1B is also important for axonal plasticity and regeneration in the adult nervous system (Nothias et al., 1996, 1997; Ma et al., 2000). In addition, MAP1B functions during development are thought to be regulated by phosphorylation, and we have previously shown that expression of a specific phosphorylated isoform (MAP1B-P) is increased in response to injury-induced axonal plasticity in the adult (Soares et al., 1998, 2002).

The present study was undertaken to further elucidate the function of MAP1B in adult axonal plasticity, particularly in regenerating axons in vitro. Thus, we analyzed axonal regeneration from cultured adult dorsal root ganglia (DRGs) neurons taken from a MAP1B null mutant mouse line able to survive several months postnatally (Meixner et al., 2000). DRGs neurons were chosen because even at adult stages they survive and regenerate their neurites in vivo and in vitro. In addition, these neurons are special in that they maintain a constitutively high expression of MAP1B in the adult (Ma et al., 1997). Finally, the peripheral axons of the mutant mice used in our study were likely to be affected by the absence of MAP1B because they exhibit a reduced diameter of myelin sheaths and a reduced conductance velocity (Meixner et al., 2000).

\section{Materials and Methods}

Animals

All experimental procedures on animals were done according to the European Community directive (86/609/EEC; authorization number 91-78 to F.N.).

Generation of MAP1B-deficient mice used in this study was described previously (Meixner et al., 2000). Briefly, these mice harbor a deletion of $93 \%$ of the MAP1B coding sequence including exons 3-5 and a part of exon 6, which leads to a complete absence of MAP1B expression. Genotyping of individual mice was done by isolating DNA from the tail, PCR amplification of a fragment spanning the homologous recombination event upstream of exon 3 of the MAP1B coding sequence was performed, and analysis of PCR products was by agarose gel electrophoresis. For the present study, we used adult ( $>2$ months of age) homozygote MAP1B knock-out mice and their control littermates (heterozygotes or wild type). As additional control, part of each cell culture prepared from homozygotes was subjected to MAP1B immunohistochemistry.

\section{Cultures of DRG explants with attached nerve stumps}

The experimental procedure essentially followed the protocol described by Tonge et al. (1997). Mice were anesthetized with Forene (Abbott, Rungis, France) and killed by decapitation. DRGs with attached distal and proximal nerve segments $(\sim 3 \mathrm{~mm}$ in length) were dissected in F-12 medium (Invitrogen, Cergy-Pontoise, France), rinsed in complete culture medium, placed on $13 \mathrm{~mm}$ coverslips, and covered with $20-30 \mu \mathrm{l}$ of growth factor reduced Matrigel (Becton Dickinson, Grenoble, France). After polymerization of the Matrigel, the preparations were covered with culture medium (RPMI 1640 supplemented with $2 \mathrm{~mm}$ L-glutamine, $100 \mathrm{u} / \mathrm{ml}$ penicillin, $100 \mu \mathrm{g} / \mathrm{ml}$ streptomycin, and $250 \mathrm{ng} / \mathrm{ml}$ Fungizone; all from Invitrogen) and incubated at $37^{\circ} \mathrm{C}, 5 \% \mathrm{CO}_{2}$ up to $6 \mathrm{~d}$.

\section{Cultures of dissociated adult DRGs neurons}

Dissected DRGs were harvested in F-12 medium, cut into small pieces before enzymatic dissociation by collagenase $\left(90 \mathrm{~min}\right.$ at $37^{\circ} \mathrm{C}, 4000 \mathrm{U} / \mathrm{ml}$; Sigma, St. Louis, MO), followed by trypsin/EDTA (Invitrogen) and
DNase 1 (50 $\mu \mathrm{g} / \mathrm{ml}$; Sigma) treatments for $10 \mathrm{~min}$ at $37^{\circ} \mathrm{C}$, and finally triturated several times through a narrowed Pasteur pipette. After washing in F-12 medium, cells were diluted in F-12 medium supplemented with N3 (Bottenstein and Sato, 1979) (all reagents were from Invitrogen), plated at a density of $\sim 100$ cells $/ \mathrm{cm}^{2}$ on precoated $13 \mathrm{~mm}$ glass coverslips, and placed in a $37^{\circ} \mathrm{C}, 5 \% \mathrm{CO}_{2}$ incubator for $12-48 \mathrm{hr}$. The coverslips were precoated with poly-L-lysine $(10 \mu \mathrm{g} / \mathrm{ml}$; Sigma) overnight at $37^{\circ} \mathrm{C}$, followed by mouse laminin $\left(10 \mu \mathrm{g} / \mathrm{ml}\right.$; at least $3 \mathrm{hr}$ at $37^{\circ} \mathrm{C}$; Sigma). In some experiments, nonpermissive stripes were created on the coverslips by removing the poly- L-lysine/laminin substrate with a sterile needle and rinsing the coverslips with sterile PBS without $\mathrm{Ca}^{2+} / \mathrm{Mg}^{2+}$.

\section{Antibody staining}

DRG cultures on coverslips were fixed in $4 \%$ paraformaldehyde/15\% sucrose in PBS for 20 min, permeabilized with PBS/0.3\% Triton X-100, and nonspecific binding sites were blocked with $10 \%$ goat serum in PBS/ $0.3 \%$ Triton X-100. For immunocytochemistry of microtubule subsets, fixation was combined with extraction in PHEM buffer (Gallo and Letourneau, 1998), $0.25 \%$ glutaraldehyde, and 0.1\% Triton X-100. Specimens were then incubated for $3 \mathrm{hr}$ with one of the following primary antibodies diluted in $\mathrm{PBS} / 5 \%$ goat serum: monoclonal anti- $\beta 3$ tubulin (Tuj-1; Babco, Richmond, CA); anti-laminin (Andujar et al., 1985); monoclonal anti-tyrosinated tubulin (Sigma); monoclonal antiacetylated tubulin (Sigma); polyclonal anti-detyrosinated tubulin (superglu) (Gundersen et al., 1984); polyclonal anti-tyrosinated tubulin $\left(\mathrm{W}^{2}\right)$ (Gundersen et al., 1984); monoclonal anti-actin (Sigma); polyclonal anti-total MAP1B (Fischer and Romano-Clarke, 1990); and FITCconjugated phalloïdin (Sigma). After several rinses in PBS/5\% goat serum, coverslips were incubated with appropriate fluorochrome-labeled secondary antibodies (Cappel/ICN, Orsay, France), rinsed again, and mounted in Mowiol.

\section{Analysis of regenerating adult DRGs neurons in vitro}

To evaluate the overall regeneration of neurites from MAP1B knock-out and wild-type DRGs, Tuj-1-immunostained cultures were analyzed on a DRBM standard light microscope (Leica, Nussloch, Germany) and photographed using a Q-imaging micropublisher camera piloted by the Q-capture system (Q-imaging, Burnaby, British Columbia, Canada). Appropriate indices were developed to compare the morphology and the regenerative capacity of neurites from knock-out versus wild-type DRGs, using ImageProPlus software (Media Cybernetics, Silver Spring, MD). All statistics were done with the ANOVA-1 test, unless indicated otherwise.

Measurement of the area occupied by neurites regenerating from $D R G$ explants. A line was drawn through the cut end of the nerve segment from which regenerating neurites emerged and then continued by joining all distal ends of the neurites. This line delineates the area occupied by axons out of the explant (Fig. 1c, schematic drawing).

Determination of rectilinear versus "curled" growth pattern of regenerating neurites. For each neurite, its actual length was measured after its real trajectory (length $b$ ), then a straight line was traced between the origin of the neurite from the explant and its distal end, representing the theoretical length of a rectilinear neurite (length a) (Fig. 1d, schematic drawing). The ratio a/b was taken as the morphological index of rectilinearity; the more this ratio is close to 1 , the more rectilinear the growth of the neurite is.

Total length of neurites growing out from dissociated DRG neurons. Both the overall length of all neurite branches emerging from a given neuron and the length of the longest neurite from the cell body to its distal end were traced and measured.

Number of branching points per main neurites growing from a dissociated $D R G$ neuron. On the neuritic tree of a given DRG neuron, the total number of branches exceeding $20 \mu \mathrm{m}$ in length was counted and divided by the number of "main" neurites (i.e., those emerging directly from the cell body). The mean of this ratio allows for an estimation of the number of branching points per main neurite.

Quantitative analysis of the behavior of growth cones facing a stripe devoid of permissive (laminin) substrate. On a photograph of the culture of dissociated DRG neurons, a band of the width of a growth cone $(\sim 10$ $\mu \mathrm{m}$ ) was delineated on the laminin substrate, adjacent to the border of 

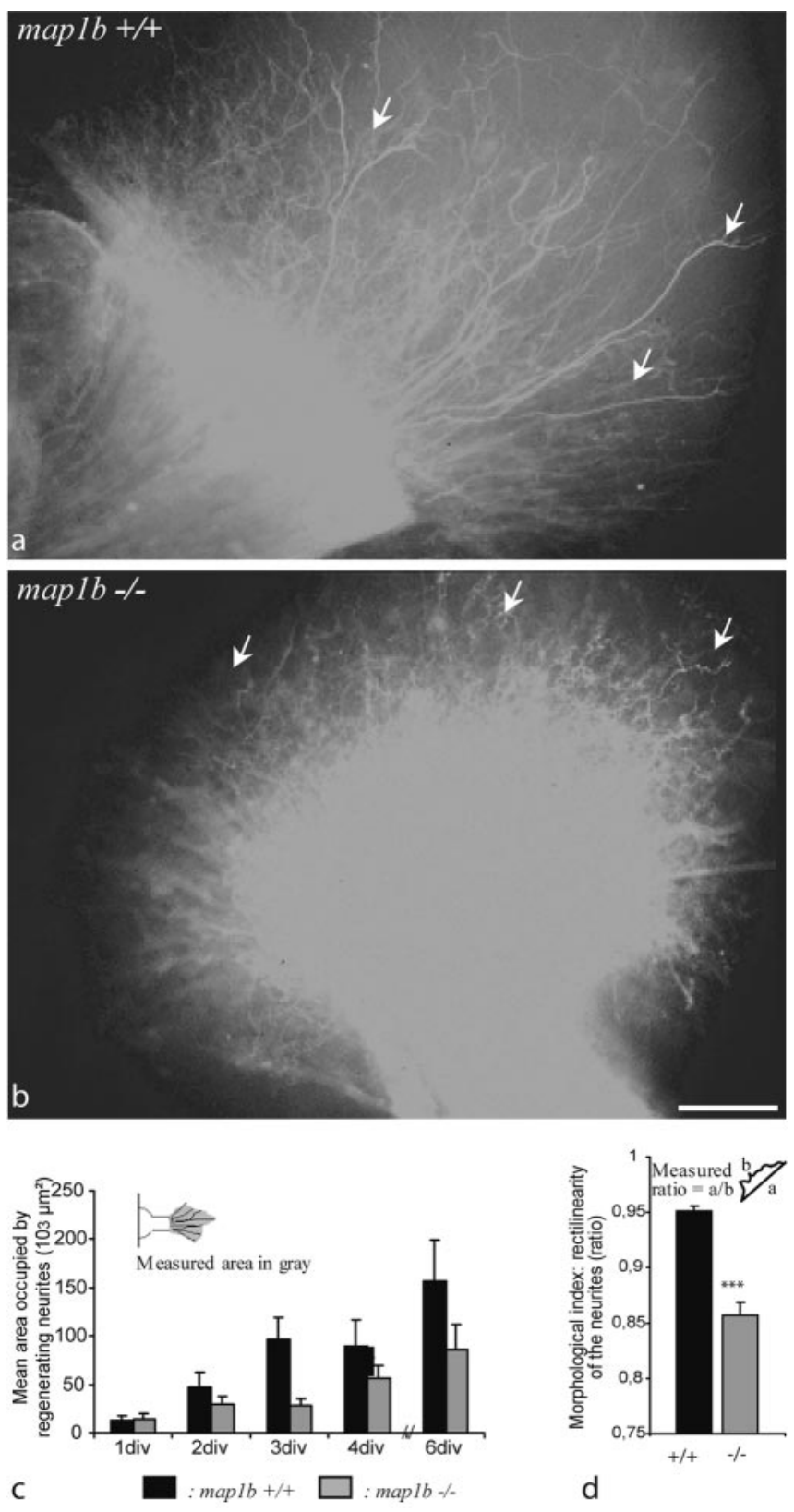

Figure 1. Axonal regrowth from adult map $1 b+/+$ and map $1 b-/-$ mice DRG explants in Matrigel. $a, b$, Immunocytochemical staining for tubulin (Tuj-1 antibody) of map $1 b+/+(a)$ and map $1 b-/-$ neurites $(b)$ after $2 \mathrm{~d}$ in vitro. Note that the growth pattern of neurites regenerating from map $16-/-$ DRG appears disoriented and curled (arrows), in contrast to the rather rectilinear growth of map $1 b+/+$ neurites (arrows). Scale bar, $200 \mu \mathrm{m}$. c, Mean area occupied by DRG neurites after $1-6 \mathrm{~d}$ of growth in vitro (measured as indicated in schematic diagram). Except for the first day, this value is consistently higher in wild-type than in MAP1B knock-out cultures. $d$, Rectilinear versus curly growth of regenerating neurites. Rectilinearity is represented as the mean ratio between actual neurite lengths and the lengths of straight lines drawn from their emergence point to the tip (as indicated in the schematic diagram). The mostly straight-growing wild-type neurites exhibit an index close to 1 , which is significantly higher than that of MAP1B knock-out neurites $\left({ }^{* * *} p<0.001\right.$; ANOVA-1), suggesting a correlation between expression of MAP1B and growth orientation.

the stripes devoid of laminin. Growth cones present in this area were counted and classified as either "stopped," if the angle between the axon axis and the nonpermissive stripe was over $45^{\circ}$, or as "turning," if this angle was below $45^{\circ}$. The relative numbers of stopped and turning growth cones in cultures of map $1 b-/-$ and map $1 b+/+$ DRGs were compared using a $t$ test.
Time-lapse video microscopy

For time-lapse video microscopy, dissociated DRGs were cultured as above on $22 \mathrm{~mm}$ laminin-precoated glass coverslips. Fifteen hours after plating, the medium was supplemented with $10 \mathrm{~mm}$ HEPES, $\mathrm{pH}$ 7.4, and cultures were placed in a chamber maintained at $37^{\circ} \mathrm{C}$ on the stage of an inverted microscope (Leica DMIRE2), equipped with differential interference contrast optics and a Plan Apo 40×/0.85 numerical aperture (NA) lens.

Still images of living neurites (500 msec) were acquired during 90-340 min at a frequency of one per minute, via a Princeton Instruments $\mathrm{Mi}-$ cromax $5 \mathrm{MHz}$ cooled CCD camera (Roper Scientific, Lisses, France). Acquisition and illumination devices were driven by MetaMorph software (Universal Imaging, West Chester, PA). Finally, sequences of 16 bit images were analyzed using MetaMorph and NIH Image J software.

Quantitative analysis of the relative amount of microtubule subsets in the growth cones

Images of MT subset staining on growth cones of DRG neurons dissociated from map $1 b+/+$ and map $1 b-/-$ mice were acquired at high resolution (Plan $100 \times / 1.3 \mathrm{NA}$ oil-immersion objective) using identical camera settings. Quantification was done on raw, untreated images of the following double staining experiments: tyrosinated MTs (polyclonal $\mathrm{W}^{2}$, FITC)/acetylated MTs (monoclonal, Cy3); detyrosinated MTs (polyclonal superglu, FITC)/acetylated MTs (monoclonal, Cy3); detyrosinated MTs (polyclonal superglu, FITC)/tyrosinated MTs (monoclonal, $\mathrm{Cy} 3)$. For each axon, an area including the growth cone and the dista part of the axon shaft ( $\sim 15-20 \mu \mathrm{m}$ from the growth cone tip) was outlined, and relative immunoreactivities (sum of red versus green pixel intensities within the defined area) quantified using Image Pro Plus software (Media Cybernetics). The ratios of Cy3/FITC fluorescence intensities were then calculated for at least 40 growth cones per condition and compared between map $1 b+/+$ and map $1 b-/-$ growth cones using an ANOVA-1 test.

\section{Results}

The present study uses a combination of two previously described in vitro models that both present specific advantages (i.e., explants of adult DRGs with nerve stumps attached) (Tonge et al., 1997) and dissociated adult DRG neurons (Lindsay, 1988). Thus, in explant cultures, the enzymatic and mechanical treatments necessary to dissociate DRGs into single cells are avoided, and the relationships between neurons, satellite cells, and Schwann cells are preserved. In addition, DRGs explants cultured in Matrigel regenerate their axons within a three-dimensional substrate, allowing for neurite-matrix interactions that may closely resemble those occurring in a natural environment. In contrast, the use of dissociated neurons on a homogeneous, twodimensional substrate (laminin) appears advantageous for the fine analysis of the morphology and the behavior of individual neurons and their corresponding neurites. It should be mentioned that DRG explants and dissociated neurons differ in one important respect that should not be neglected in axonal regeneration studies: dissection of DRG explants leaves the proximal part of the neurites intact, whereas dissociation of DRG neurons involves a loss of all cell processes. Thus, re-elongation of preexisting axons, and initiation of new neurites emerging from the cell body, may rely on slightly different molecular mechanisms.

\section{Lack of MAP1B affects the growth pattern of DRG axons regenerating ex vivo}

DRG explants with dorsal roots and peripheral nerve attached were prepared from adult map $1 b+/+$ and map $1 b-/-$ mice and kept in culture for $1-6 \mathrm{~d}$. For map $1 b+/+$ explants, results were comparable with those described previously (Tonge et al., 1997). Within a few hours, thin neurites started to extend from the cut nerve stumps into the Matrigel. Outgrowth of regenerating axons 
continued for $\sim 1$ week (Fig. 1a, $2 \mathrm{~d}$ in vitro), before the growth rate decreased and the axons started to degenerate.

In preliminary experiments, we observed that neurites regenerating from the peripheral nerve stump were mostly longer than those growing from the dorsal root (see also Tonge et al., 1996). Thus, the following comparative analysis between wild-type and MAP1B-deficient DRG explants is based on fibers growing from the peripheral nerve.

At first sight, neurites regenerate from both map $1 b+/+$ and map $1 b-/-$ DRG explants (Fig. 1, compare $a, b$ ). Thus, extension of regenerating axons into the Matrigel followed about the same time course as in the control experiments, with regard to both the initiation of neurite growth and mean neurite survival time. Nevertheless, the extending axons exhibited greater variations in length, indicating that regeneration from map $1 b-/-$ explants may be less robust. We then analyzed various parameters that allowed for a more precise comparison between axon regrowth from maplb-/- versus control DRGs. First, we measured the total surface occupied by regenerating neurites (Fig. 1c). Indeed, the mean area, occupied by regenerating axons, turned out to be different between map $1 b-/-$ and map $1 b+/+$ DRGs, and this difference was increasing with time: at the end of a 6 d culture period, neurites growing from map $1 b+/+$ DRGs covered an area twice as large as those growing from map $1 b-/-$ DRGs.

This could, in principle, be attributable to a rate of neurite extension that would be lower for axons lacking MAP1B. Because of the high density of axons, which moreover grow in different focal planes within the Matrigel, this aspect was difficult to analyze in DRG explants but was addressed using cultures of dissociated DRG neurons (see below). A second explanation might be related to the growth pattern of individual axons. Thus, we noticed that the final shape of axons that lacked MAP1B was generally frizzy/wavy, whereas map $1 b+/+$ DRG axons appeared smooth and rectilinear. We were able to quantify this aspect by introducing a "rectilinearity" index for growing neurites (i.e., the ratio between the overall length of a more or less meandering neurite) and the distance of the growth cone from the nerve stump (see Materials and Methods). The more this index is close to 1 , the more the neurites are rectilinear. Because this parameter did not change over the culture period, we were able to pool the data obtained at different time points for each population of regenerating neurites. A quantification is shown in Figure 1d, indicating a significant difference between the growth patterns of map $1 b-/-$ versus map $1 b+/+$ neurites $(p<0.001$; ANOVA- 1$)$. Indeed, map $1 b+/+$ neurites grew almost straightforward (mean, $0.95)$ compared with a more wavy growth of map $1 b-/-$ neurites (mean, 0.86).

Together, analysis of our explant cultures shows that adult DRG neurons, placed in an environment favorable for axon growth, are able to regenerate their axons despite the absence of MAP1B, but their outgrowth pattern is affected.

\section{Branching, rather than elongation capacity, is altered in axons regenerating from map $1 b-/-$ DRGs}

When plated on a uniform, growth-permissive laminin substrate, dissociated adult DRG neurons started to elaborate processes after only a few hours in culture. During $48 \mathrm{hr}$, the neuritic tree kept growing and got more and more complex. Thereafter, degenerative processes would start to appear. As observed with explant cultures, most regenerating map $1 b+/+$ neurites had a straight orientation and finally occupied a large area on the substrate; their elongation was most intense between 12 and $24 \mathrm{hr}$ in vitro (Fig. 2a,c). In contrast, neurites from map1b-/- DRGs again
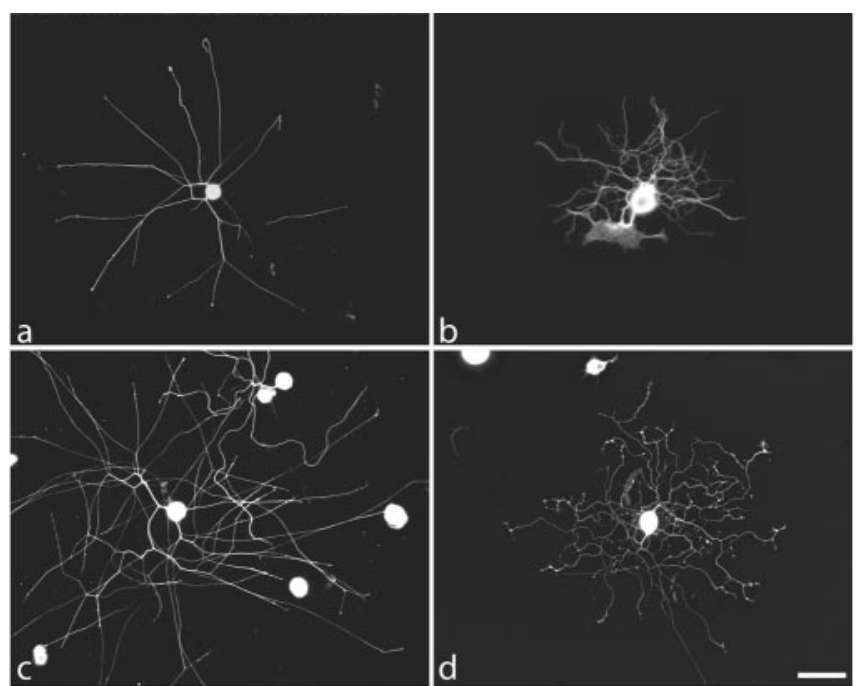

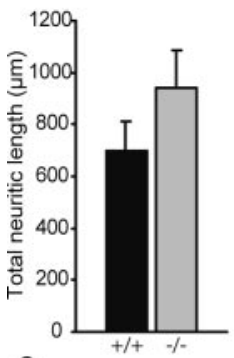

e
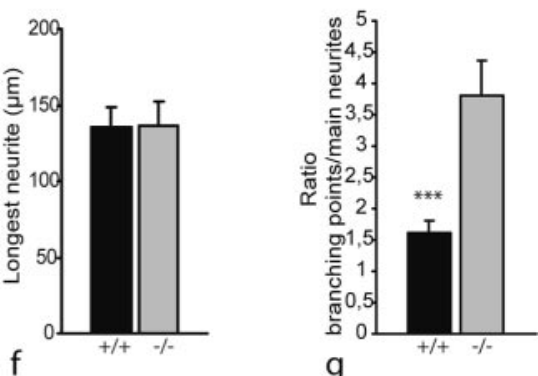

Figure 2. Morphology of neurites regenerating from dissociated wild-type $(a, c)$ and map $1 b-/-(b, d)$ DRG neurons after $12 \mathrm{hr}(a, b)$ and $24 \mathrm{hr}(c, d)$ in vitro, visualized by tubulin immunostaining (Tuj-1 antibody). As observed in DRG explant cultures, neurites lacking MAP1B appear more curled than wild-type neurites. Note also the high number of branching points present on map $1 b-/-$ neurons. Scale bar, $100 \mu \mathrm{m} . e-g$, Quantitative analysis after $12 \mathrm{hr}$ in vitro of total length of neuritic trees $(e)$ and length of the longest neurite $(f)$. No significant difference was observed between cultures of map $1 b-/-$ and map $1 b+/+$ neurons. $g$, In contrast, the number of branching points per main neurite is significantly higher for map $1 b-/-$ than for map $1 b+/+$ neurons $\left({ }^{* * *} p<0.001\right.$; ANOVA- 1 ).

exhibited a curled aspect, as observed previously in explant cultures (Figs. $1 b, 2 b, d$ ). Neuritic extension also occurred within the first $24 \mathrm{hr}$, but the area occupied by these axons appeared smaller than for map $1 b+/+$ neurons (Fig. 2). However, a quantitative analysis (Fig. 2e,f) revealed no significant differences in the average length of map $1 b+/+$ versus map $1 b-/-$ DRG neurites, in the total length of the neuritic tree, or the length of the longest neurite per individual neuron (the latter index may reflect the polarization of a neuron, which may be different in vitro).

Thus, our results indicate that the lack of MAP1B affects the elaboration of the morphology of regenerating neurites, rather than their extension rate. This was most obvious when analyzing the number of branching points per neurite, which was systematically higher for map $1 b-/-$ neuron cultures. Quantitative analysis of the mean number of branching points per main neurite yielded a twofold higher value for map $1 b-/-$ than for map $1 b+/+$ neurites (Fig. $2 g$ ).

Together, although the average length of regenerating neurites from map $1 b+/+$ and map $1 b-/-$ DRGs is not significantly different, the surface covered by DRG neurites from mutant mice is smaller because of the high number of branching points, in combination with the curled aspect of these neurites.

To assess the dynamic aspect of branch elaboration on neu- 
rites regenerating from map $1 b-/-$ in comparison with wild-type neurons, live cultures of dissociated DRGs were analyzed using time-lapse video microscopy. Movies were composed of sequential 500 msec still images of growth cones and neurite branching points, taken at 1 min intervals over periods of $90-340 \mathrm{~min}(n=4$ for map $1 b-/-$ and $n=4$ for map $1 b+/+$ neurons), and images were also analyzed frame by frame. To illustrate the dynamics of growth cones during the process of branch formation, Figure 3 shows examples of images acquired in $10 \mathrm{~min}$ intervals for a period of $90 \mathrm{~min}$. Both map $1 b+/+$ and map $1 b-/-$ growth cones are very dynamic structures, extending and retracting their filopodia (Fig. 3 and see movies, available at www.jneurosci.org/cgi/content/ full/24/32/7204/DC1). In somecases, a growth cone was seen to bifurcate, and each of the two branches continued to elongate (Fig. $3 a, b)$. However, this phenomenon was more frequent in map $1 b-/-$ cultures (Fig. $3 b$ ). The majority of map $1 b+/+$ neurites progressed by continuous elongation in the orientation of a given, individual filopodium, whereas others were retracted (Fig. 3c). Another obvious difference between mutant and wild-type neurites concerned the elaboration of proximal branching points (i.e., far behind the terminal growth cone). Very often, map $1 b-/-$ neurites were seen to exhibit an enlargement or swelling along their shafts, followed by the appearance of filopodia-like protrusions that are eventually transformed into a new collateral branch (Fig. $3 d$, sequence). In contrast, small filopodia-like extensions that might form along the shafts of map $1 b+/+$ neurites are, in most cases, retracted, so that wild-type neurites exhibit a less branched, straighter appearance (Fig. 3a,c).

Thus, our time-lapse video microscopy analysis confirmed the results obtained from fixed cultures of DRG neurons, demonstrating that branching is more frequent on map1b-/- neurites than on their map $1 b+/+$ counterparts. In addition, the analysis of live cultures showed that although both map1b+/+ and map1b-/neurites are able to elaborate terminal branches, the extensively branched aspect of map $1 b-/-$ neurites can in part be attributed to collaterals forming along the neurite shaft.

\section{Choice of growth direction is impaired in growth cones lacking MAP1B}

The experiments described above suggested that the lack of MAP1B might affect the directionality of DRG neurites regen-
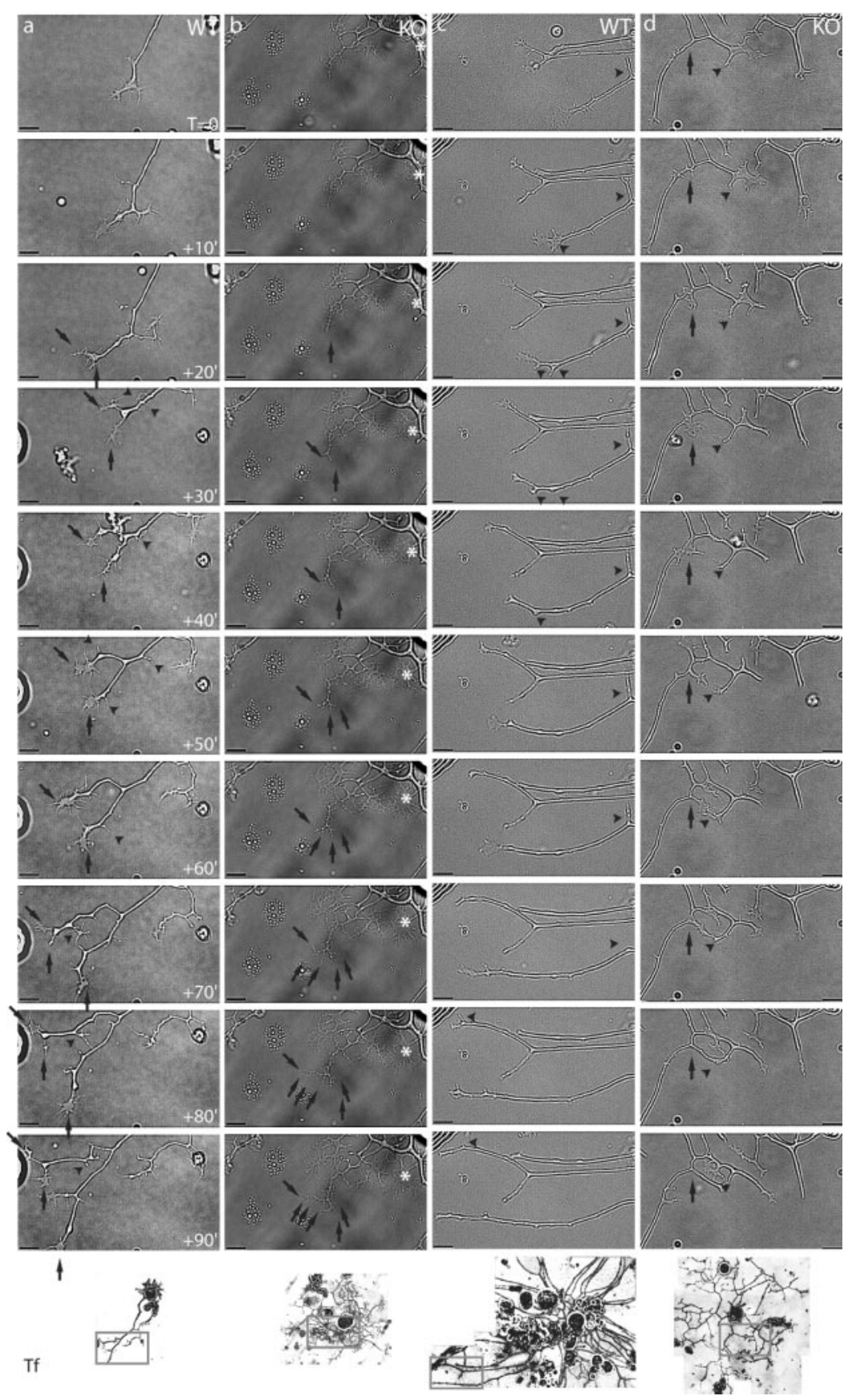

Figure 3. Time-lapse video microscopy images of neurites regenerating from dissociated wild-type (WT) and map $16-/-$ (K0) DRG neurons. The four 90 min sequences represent the evolution of individual neurites after an initial culture period of $15-18 \mathrm{hr}$ (set as To), in 10 min intervals between frames. An overview of each neuron at the end of the acquisition time is presented at the bottom (Tf). Scale bar, $10 \mu \mathrm{m} . a$, Terminal branching on a map $1 b+/+$ neurite. After neurite extension for the first $20 \mathrm{~min}$, two particularly long filopodia are distinguished on the growth cone that will give rise to two new, separately extending branches (arrows). After 60 min, new filopodia emerge from the upper growth cone, which after two separate branching events give rise to three new processes. Two of them are stabilized (arrows), and the third one retracts (arrowhead). Frequent retraction of unstabilized new processes leads to a straight aspect of wild-type neurites (arrowheads; see To $+90^{\prime}$ and panel Tf). $b$, Terminal branching and filopodia dynamics on map $1 b-/-$ neurites. The neurite shown presents numerous thin, filopodia-like processes and a particularly large growth cone (asterisk) with several actively extending and retracting filopodia. In a sequence of terminal branching events between $\mathrm{T}_{0}+30^{\prime}$ and $\mathrm{T}_{0}+90^{\prime}$, numerous new processes are elaborated (arrows) that, in contrast to map $1 b+/+$ neurons, do not retract, eventually giving rise to the highly branched aspect of the map $1 b-/-$ neuron. c, Three smooth, straight neurites extending from the same map $1 b+/+$ neuron. Their growth cones are small, typical for rapidly extending neurites. Thin, short new processes forming by growth cone bifurcation are always retracted (arrowheads), so that the final morphology of all three neurites remains rectilinear and smooth (Tf). $d$, Collateral branching events on a map $1 b-/-$ neurite. At $\mathrm{To}$, a proximal segment on the neurite shaft (arrow) undergoes a swelling, followed by the appearance of filopodia ( $\mathrm{T}_{0}+10^{\prime}$ ) that finally give rise to a new growth cone $\left(\mathrm{T}_{0}+30^{\prime}\right)$. During extension of this new growth cone, it comes into contact with another process (arrowhead), originating from a previous terminal branching event $\left(\mathrm{T}_{0}+60^{\prime}\right)$. This latter process then retracts, whereas the new collateral branch is obviously stabilized and continues to progress ( $\mathrm{T}_{0}+90^{\prime}$; see also $\mathrm{Tf} 2.5 \mathrm{hr}$ later). 

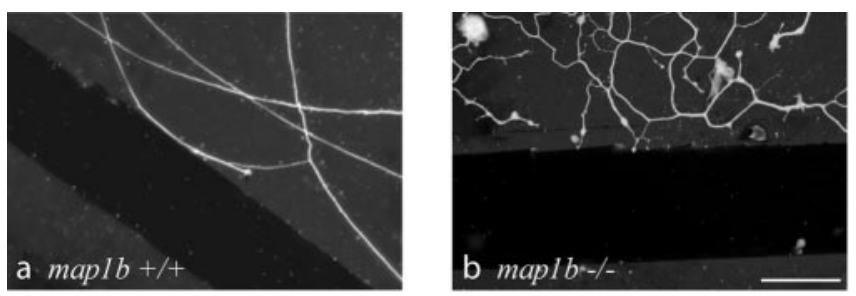

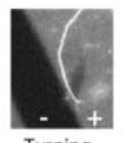

Turning

C

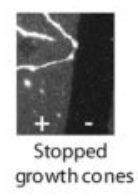

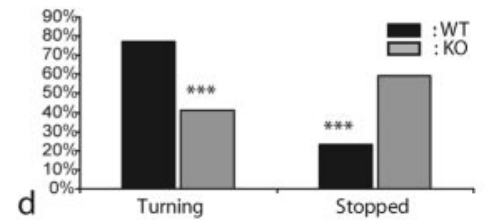

Figure 4. Turning behavior of growth cones approaching a stripe devoid of permissive (laminin) substrate. Neurites of dissociated adult map $1 b+/+(a)$ and map $1 b-/-(b)$ DRG neurons were grown for $2 \mathrm{~d}$ on a laminin substrate (gray background), on which nonpermissive stripes were created by scratching off the laminin (dark bands). Neurites are visualized by immunofluorescence staining for tubulin (Tuj-1 antibody). Scale bar, $50 \mu \mathrm{m}$. $a$, typical example of a map $1 b+/+$ growth cone that turns and continues its outgrowth after facing the nonpermissive substrate. The angle between a turning neurite and the nonpermissive band is usually $<45^{\circ}$. $b$, In the same situation, a majority of map $1 b-/-$ growth cones is stopped, exhibiting an angle of $>45^{\circ}$ to the nonpermissive band. $c$, Both behaviors in higher magnification. $d$, Quantification of the number of stopped and turning growth cones, in relation to the neuron genotype. The difference between map $1 b+/+(n=137)$ and map $1 b-/-$ neurons $(n=97)$ is significant ${ }^{* * *} p<0.001 ; t$ test).

erating on a uniform two- or three-dimensional substrate. To verify this hypothesis, we set up an in vitro assay aimed at analyzing the behavior of growth cones confronted with a situation that would force them to change direction. Thus, dissociated adult DRG neurons were seeded on laminin-coated coverslips, on which non-growth-permissive stripes had been created by scratching off the substrate with a needle (Fig. $4 a-c$ ).

In these cultures, two types of responses were observed when growth cones approached a region devoid of laminin substrate: the growth cone either turned, and neurite extension continued in a direction parallel to the nonpermissive band, or the growth cone would stop, and neurite extension was blocked (Fig. 4c). Quantification of the behavior of growth cones from map $1 b+/+$ and map $1 b-/-$ DRGs yielded the following result: a large majority of map $1 b+/+$ growth cones $(>75 \%)$ turned away from the nonpermissive stripe and continued progression on the laminin substrate (Fig. $4 a, d$ ). In contrast, $60 \%$ of the neurites lacking MAP1B were blocked when their growth cone came in contact with the nonpermissive stripe (Fig. $4 b, d$ ). This difference in the behavior of growth cones from map $1 b+/+$ versus map $1 b-/-$ DRG neurons facing a nonpermissive substrate is significant $(p<0.001 ; t$ test $)$.

Together, our study demonstrates that lack of MAP1B indeed affects the regeneration of adult DRG neurons in vitro. Because MAP1B is likely to be implicated in regeneration-induced reorganization of the cytoskeleton, we used dissociated adult DRG neurons to perform an immunohistochemical analysis of the distribution and organization of microtubule subsets and AFs.

\section{Organization of the microtubule cytoskeleton in regenerating DRG neurites}

The transition from a highly dynamic to a more stable state of microtubules is accompanied by post-translational modifications on the tubulin polymers (see Discussion). Thus, C-terminal detyrosination and/or acetylation, occurring independently on different sites on the $\alpha$-tubulin molecule, appear to be character- istic of MTs that display a low turnover rate and that are more resistant to drug-induced depolymerization (Schulze et al., 1987; Webster and Borisy, 1989). Accordingly, detyrosinated/acetylated MTs are predominantly found in the axon shaft, whereas tyrosinated (unmodified) MTs are enriched toward the distal end of the axon and within the growth cone (Baas and Black, 1990; Ahmad et al., 1993; Brown et al., 1993). Here, we used specific antibodies to determine the distribution and evaluate the relative amount of both modified and unmodified MTs in regenerating DRG neurites growing on a homogeneous laminin substrate.

Control map $1 b+/+$ DRG neurites displayed the well documented, "normal" distribution of tyrosinated versus detyrosinated/acetylated MTs (Robson and Burgoyne, 1989; Baas et al., 1991; Brown et al., 1993). As seen in Figure 5, $b$ and $f$, intensely stained bundles of tyrosinated MTs extended far into the growth cone C-domain. Detyrosinated and acetylated MTs were also observed up to the C-domain of growth cones, but the distal decrease in staining intensity was sharper than for tyrosinated MTs (Fig. $5 b, d, f$ ).

In DRG neurites derived from homozygous map $1 b-/-$ mutants, the distribution of tyrosinated and detyrosinated MTs was similar to wild-type neurites (Fig. 5c,e,g). Acetylated MTs, however, appeared to be much less prominent in the distal axon shafts and growth cones of map1b-/-, than of wild-type neurites (Fig. 5 , compare $b, c$ with $d, e$ ). To quantify the relative ratios of tyrosinated, detyrosinated, and acetylated microtubule subsets in wildtype versus map $1 b-/-$ neurites, we performed a series of double-staining experiments with different combinations of these three markers (see Materials and Methods and Fig. 5). Experiments were evaluated by measuring staining intensities using the Image Pro Plus program and calculating the ratios between relative amounts of two MT subsets, thus providing an internal standard for each measurement. Confirming our qualitative observations, Figure $5 a$ shows that indeed the staining level of acetylated tubulin was significantly lower in map $1 b-/-$ than in wildtype growth cones. In contrast, no significant difference was seen for tyrosinated and detyrosinated MTs.

We also examined the distribution of AFs in neurites regenerating from map $1 b-/-$ and map $1 b+/+$ DRG neurons, but no apparent difference was noted (data not shown).

Finally, because the distribution of MAP1B had been essentially described for developing neurons [from our own studies and those of Black et al. (1994), Boyne et al. (1995), and Bush et al. (1996)], we also performed MAP1B immunostaining on regenerating neurites. As during development, MAP1B was enriched toward the distal part of growing neurites, in growth cones, and at branching points (Fig. 6). The overall distribution of MAP1B reflected that of tyrosinated MTs, and the colocalization of these two cytoskeletal proteins is illustrated in Figure 6, $a$ and $c$. Occasionally, the presence of MAP1B extended as far as the P-domain, where colocalization with AFs could be observed (Fig. $6 c$ ). Figure $6 b$ further shows that at neurite regions exhibiting a high F-actin content (e.g., branching points), the MAP1B staining pattern would change from a bundled to a splayed aspect, as described previously for MTs themselves (Gallo and Letourneau, 1998).

\section{Discussion}

Several recent studies, mainly from our laboratory, demonstrated that MAP1B expression is associated with axonal plasticity in the adult brain (Nothias et al., 1996, 1997). We also showed that MAP1B expression in adult DRG neurons is maintained at levels similar to those of developing DRGs (Ma et al., 1997), suggesting that this protein might be among the neuron-intrinsic factors 
responsible for the plastic and regenerative capacity of adult DRG neurons. Furthermore, we presented evidence for the induction of a specific phosphorylated isoform of MAP1B in axons undergoing injury-induced plasticity (Soares et al., 2002).

The present study addresses the question of the function of MAP1B in regenerating axons. We demonstrate that the regenerative capacity of adult DRGs per se is not impeded in the absence of MAP1B. However, we reveal certain abnormalities in the regulation of collateral formation and in growth directionality of the neurites (i.e., in the capacity of their growth cones to turn when facing a nonpermissive substrate).

\section{Adult map1b-/- DRG neurons do initiate and elongate neurites in vitro}

We show here that neither initiation nor elongation of neurites regenerating from adult DRG neurons seemed to be affected by the lack of MAP1B. Our results differ from those provided by Gonzalez-Billault et al. (2002), who reported a reduced axon elongation, most likely caused by an inhibition in the early stages of axogenesis. It is important to note that this latter study used DRG cultures derived not from adult but from embryonic day 18 embryos. In addition, this MAP1B mutant is a hypomorph, still expresses low levels of MAP1B, and has a strong developmental phenotype leading to perinatal death of homozygotes. The reasons for this unusually strong phenotype that is found neither in another hypomorph (Takei et al., 1997) nor in the MAP1B null mice used here (Meixner et al., 2000) are not clear but could be attributable to influences of the genetic background. To avoid potential problems arising from residual MAP1B expression in hypomorphic mutants, we decided to investigate DRG neuron regeneration in MAP1B null mutant mice.

\section{MAP1B regulates branching during axonal regeneration}

The outgrowth pattern of neurites regenerating from map $1 b-/-$ DRGs was strikingly different from that of wild-type DRGs. Neurites would often grow in a curled manner and exhibit twice as many collateral branches than wild-type neurites. This observation strongly suggests an implication of MAP1B in the regulation of axonal branching.

Formation of new branches generally occurs on pausing, spread growth cones and involves reorganization of both MTs a.
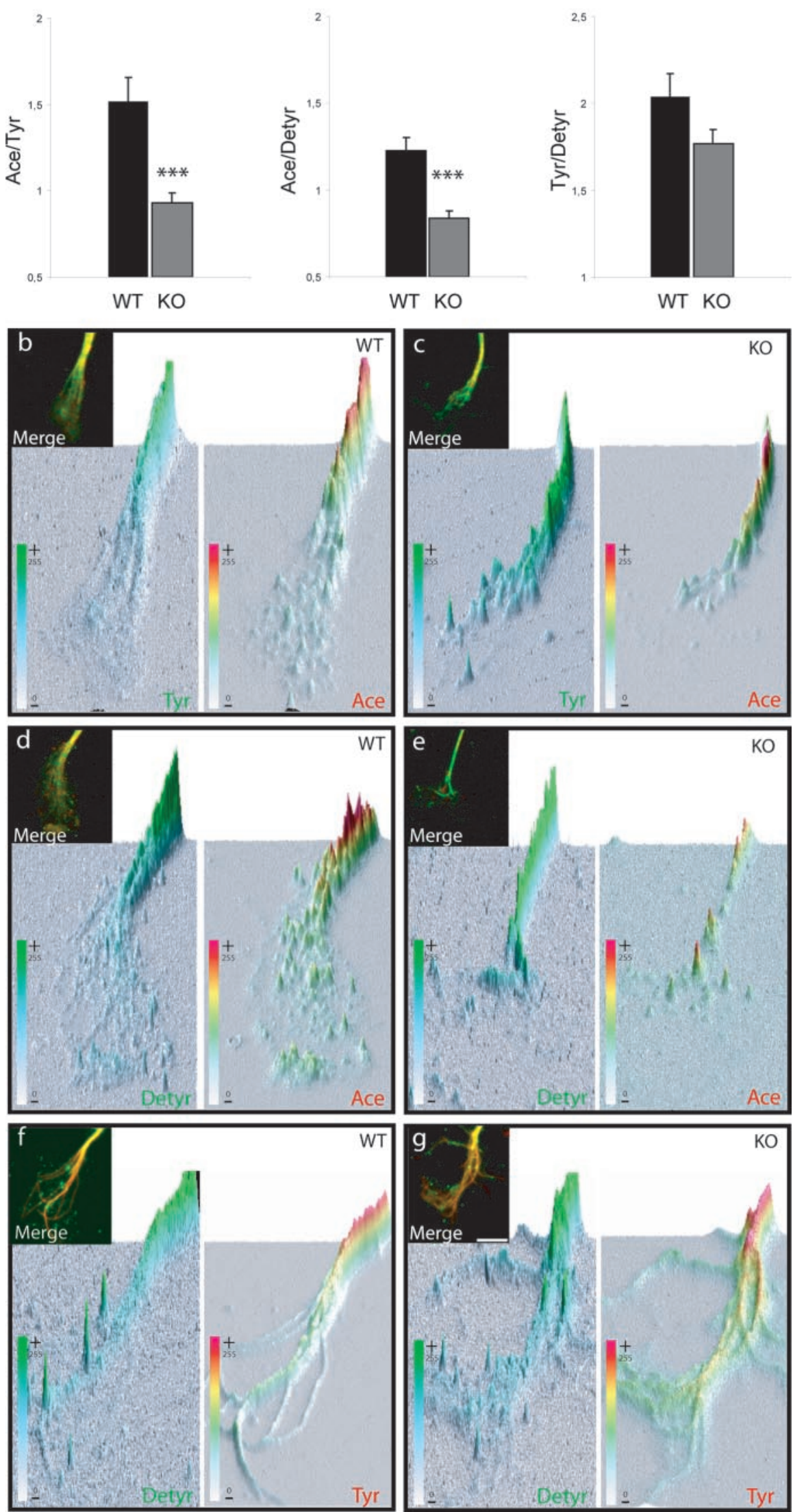

Figure 5. map $1 b-/-$ growth cones display reduced relative amounts of acetylated tubulin immunostaining. $a$, Immunofluorescence ratios (arbitrary units) were calculated for map $1 b+/+$ [wild-type (WT)] and map $1 b-/-$ [knock-out (K0)] growth cones between, acetylated and tyrosinated tubulin (Ace/Tyr), acetylated and detyrosinated tubulin (Figure legend continues.) 

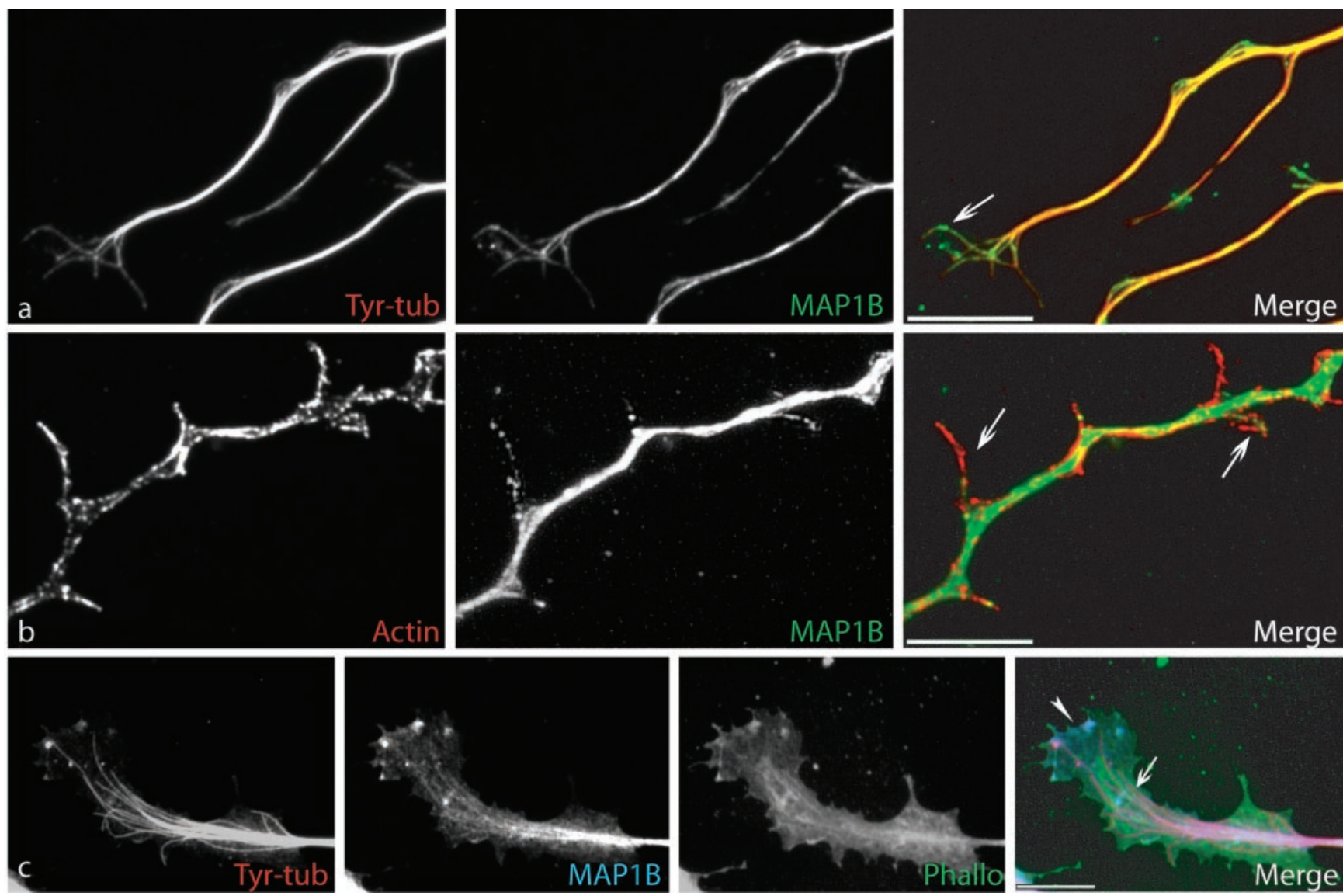

Figure 6. MAP1B immunolocalization in growth cones and at branching points. a, Double immunostaining for tyrosinated MTs (Tyr-tub; red) and MAP1B (green) shows that both proteins colocalize along the axon shaft and in the growth cone, in which individual MT bundles extend into the P-domain (arrow). Note the splayed aspect of both cytoskeletal components in the distal region and at branching points. $b$, Double immunostaining with anti-actin (red) and MAP1B (green) antibodies reveals colocalization of both proteins along the neurites in filopodia and newly formed branches (arrows). c, Triple immunostaining for tyr-tubulin (red), MAP1B (blue), and actin-phalloidin (green), demonstrating the colocalization of MAP1B with tyrosinated MTs (pink in merge panel; arrow) in the growth cone and with AFs (light blue; arrowhead) in the P-domain. Scale bar, $10 \mu \mathrm{m}$.

and AFs. Within the resting growth cone, F-actin is concentrated in distal regions in which the MTs splay apart. Branching starts with an individual filopodium, originally containing mostly F-actin and few tyrosinated microtubules, by coordinated polymerization of both actin and tubulin. The former filopodium then elongates and finally behaves like a new axon (Challacombe et al., 1997). In addition to the organization of MTs and F-actin, we show here that MAP1B exhibits the same distribution as tyrosinated MTs. Using time-lapse video microscopy, we were able to observe the sequential steps of branch elaboration at the distal end of growing neurites, both on map1b+/+ and map1b-/DRG cultures. However, in map $1 b-/-$ DRG cultures, formation of collateral branches along the proximal part was also frequent, reflecting a highly dynamic neurite shaft.

Although axon growth at the distal end and collateral sprouting might rely on different mechanisms (Gallo and Letourneau, 1998, 1999), the terminal growth cone has been reported to pause and enlarge in certain "active" regions in which later interstitial axon branches may develop (Szebenyi et al., 1998, 2001). In such regions, numerous short, filopodia-like processes appear along the neurite shaft, most of which are transient and do not form growth cones (Szebenyi et al., 1998). In cultures of map1b-/DRG neurons, however, collateral branches did not systematically emerge from preexisting filopodia. The neurite shaft was seen to undergo swelling, followed by the protrusion and retraction of several filopodia-like spines, one of which would eventually develop into a growing collateral that often persisted for the entire observation period. Our results suggest that branching of map $1 b-/-$ neurites is not restricted to an active region and that neurite shafts may remain especially dynamic.

To further analyze the relationship between lack of MAP1B and microtubule dynamics, we evaluated the relative abundance of the different MT subsets in the distal part of the axon shaft and in the growth cone using specific antibodies to post-translationally modified and unmodified tubulin polymers as indirect markers for MT stability. In fact, it is now well established that highly dynamic MT polymers, while present throughout the axon, are particularly frequent in the distal portion and the growth cone (Ahmad et al., 1993). Stable and labile MT polymers differ in their rate of drug-induced depolymerization, solubility properties, and subunit turn-
(Figure legend continued.) (Ace/Detyr), and tyrosinated and detyrosinated tubulin (Tyr/Detyr). Quantitative and statistical analysis shows that both ratio Ace/Tyr and Ace/Detyr are significantly lower in map $1 b-/-$ than map $1 b+/+$ growth cones, whereas the Tyr/Detyr ratio is not significantly different between the two genotypes. ${ }^{* * *} p<0.001 ; \mathrm{ANOVA-1;} n=40$ for each condition. $b-g$ Example of double immunofluorescence of map $16+/+$ (WT) and map $1 b-/-$ (K0) growth cones for each condition presented in the diagrams in $a$. The insets show examples of original merged images of double-immunostained growth cones, from which three-dimensional graphs were created (Image Pro Plus program), representing the spatial distribution of individual colors in the growth cone and their pixel intensities on the $z$-axis (also color-coded). Scale bar, $5 \mu \mathrm{m}$. 
over rate. The labile domain is more sensitive to drug-induced depolymerization and contains relatively high amounts of tyrosinated $\alpha$-tubulin (Baas and Black, 1990; Ahmad et al., 1993). Stable MTs, more resistant to depolymerizing drugs, are usually enriched in acetylated and/or detyrosinated tubulin (Thompson et al., 1984; Kreis, 1987; Piperno et al., 1987; Schulze et al., 1987; Webster et al., 1987; Bulinski et al., 1988; Baas and Black, 1990; Ahmad et al., 1993; Matsuyama et al., 2002), although MTs post-translational modifications are not themselves the cause of MT stabilization (Webster et al., 1990).

Interestingly, acetylated MTs were less abundant in distal regions of map $1 b-/-$, than of map $1 b+/+$ neurites, whereas tyrosinated or detyrosinated MTs appeared not affected. This is in agreement with previous data demonstrating that MAP1B transfection into non-neuronal cells induces an increase in acetylated MTs (Takemura et al., 1992). Although MAP1B itself has a higher affinity for tyrosinated MTs (Goold et al., 1999), it is clearly involved in MT stabilization, as are the other MAPs (Takemura et al., 1992; Tögel et al., 1998). Thus, a decrease in the amount of acteylated MTs similar to that described here could also be evoked by suppression of tau (Caceres et al., 1992).

Additional studies will be necessary to elucidate the causal relationship between decreased levels of acetylated MTs in the absence of MAP1B and the increased rate of collateral formation. Increased branching has also been reported for CNS neurons deficient in the kinesin superfamily protein KIF2A (Homma et al., 2003). These authors suggest that KIF2A has an MTdepolymerizing activity responsible for the suppression of collateral branch extension. At first sight, this latter mechanism may seem contradictory to the one proposed here (i.e., inhibition of collateral formation by stabilization of axonal microtubules, accelerated in the presence of MAP1B). However, MAP1B deficiency is leading to collateral formation from mature axon shafts, KIF2A deficiency to stabilization, and extension of branches from the growth cone. Axonal branching obviously requires dynamic MTs that are able to grow. It is conceivable that increase of stabilized axonal MTs in the presence of MAP1B (Tögel et al., 1998) or depolymerization of MTs by KIF2A in the growth cone will reduce the pool of growing MTs and thus prevent branching.

\section{MAP1B is required for turning of regenerating axons}

The curled growth pattern of axons regenerating from map1b-/-DRG neurons, compared with the rather straightforward growth of wild-type neurites, prompted us to investigate whether lack of MAP1B might influence the capability of growth cones to "choose" a proper orientation. Indeed, one of the major findings of the present study is the direct demonstration of the implication of MAP1B in growth cone turning of regenerating axons: the majority of maplb-/- DRG neurites, although able to grow on a homogeneous permissive substrate, stopped at the limit of a band devoid of substrate. Confronted with the same situation, map $1 b+/+$ growth cones rather turned and continued to progress along the limit of the nonpermissive band. Our results are in agreement with a previous in vitro study on embryonic retinal neurons (Mack et al., 2000). These authors used chromophore-assisted laser inactivation on a microscale (microCALI) to neutralize a specific MAP1B phospho-isoform and found that, in consequence, turning of growth cones at nonpermissive substrate borders was affected. In addition, they demonstrated that the presence of phosphorylated MAP1B in a normal growth cone was restricted to sites that are stabilized and thus oriented toward a potential turning direction (for review, see Tanaka and Sabry, 1995).
From these and our results, it seems evident that the reorientation capacity of growth cones depends mostly on MAP1B and its phosphorylation state, because lack of MAP1B could obviously not be compensated by other MAPs, despite the continued presence of dynamic MTs, enriched in tyrosinated tubulin. The developmental role of MAP1B in the organization of the cross talk between MTs and AFs (Owen and Gordon-Weeks, 2003; Dehmelt and Halpain, 2004) thus appears to be maintained in adult axonal plasticity. A future objective will be to elucidate how external guidance cues may regulate the functions of MAP1B in axon branching and turning described here. In this respect, it is interesting to note that the brain of our MAP1B-deficient mice exhibits a specific developmental defect (i.e., agenesis of the corpus callosum) (Meixner et al., 2000). Thick bundles of the corresponding callosal fibers are found stalling in lower layers of the cortex, indicating that they are unable to cross the midline. MAP1B may thus be part of a signal transduction pathway guiding axons across an environment determined by midline glia cells (Meixner et al., 2000, their Discussion). Finally, we previously demonstrated (Soares et al., 2002) that injury-provoked deafferentation of a specific area in the adult spinal cord is followed by rapid induction of a phosphorylated MAP1B isoform within axons sprouting exclusively toward this area. These data suggested an implication of MAP1B in growth cone pathfinding in response to specific attractive guidance cues present in areas of deafferented postsynaptic neurons.

\section{References}

Ahmad FJ, Pienkowski TP, Baas PW (1993) Regional differences in microtubule dynamics in the axon. J Neurosci 13:856-866.

Andujar MB, Magloire H, Hartmann DJ, Ville G, Grimaud JA (1985) Early mouse molar root development: cellular changes and distribution of fibronectin, laminin and type-IV collagen. Differentiation 30:111-122.

Baas PW, Black MM (1990) Individual microtubules in the axon consist of domains that differ in both composition and stability. J Cell Biol 111:495-509.

Baas PW, Luo L (2001) Signaling at the growth cone: the scientific progeny of Cajal meet in Madrid. Neuron 32:981-984.

Baas PW, Slaughter T, Brown A, Black MM (1991) Microtubule dynamics in axons and dendrites. J Neurosci Res 30:134-153.

Black MM, Slaughter T, Fischer I (1994) Microtubule-associated protein 1b (MAP1b) is concentrated in the distal region of growing axons. J Neurosci 14:857-870.

Bottenstein JE, Sato GH (1979) Growth of a rat neuroblastoma cell line in serum-free supplemented medium. Proc Natl Acad Sci USA 76:514-517.

Boyne LJ, Martin K, Hockfield S, Fischer I (1995) Expression and distribution of phosphorylated MAP1B in growing axons of cultured hippocampal neurons. J Neurosci Res 40:439-450.

Brown A, Li Y, Slaughter T, Black MM (1993) Composite microtubules of the axon: quantitative analysis of tyrosinated and acetylated tubulin along individual axonal microtubules. J Cell Sci 104:339-352.

Bulinski JC, Richards JE, Piperno G (1988) Posttranslational modifications of alpha tubulin: detyrosination and acetylation differentiate populations of interphase microtubules in cultured cells. J Cell Biol 106:1213-1220.

Bush MS, Goold RG, Moya F, Gordon-Weeks PR (1996) An analysis of an axonal gradient of phosphorylated MAP $1 \mathrm{~B}$ in cultured rat sensory neurons. Eur J Neurosci 8:235-248.

Caceres A, Mautino J, Kosik KS (1992) Suppression of MAP2 in cultured cerebellar macroneurons inhibits minor neurite formation. Neuron 9:607-618.

Challacombe JF, Snow DM, Letourneau PC (1996) Actin filament bundles are required for microtubule reorientation during growth cone turning to avoid an inhibitory guidance cue. J Cell Sci 109:2031-2040.

Challacombe JF, Snow DM, Letourneau PC (1997) Dynamic microtubule ends are required for growth cone turning to avoid an inhibitory guidance cue. J Neurosci 17:3085-3095.

Dehmelt L, Halpain S (2004) Actin and microtubules in neurite initiation: are MAPs the missing link? J Neurobiol 58:18-33.

Dent EW, Gertler FB (2003) Cytoskeletal dynamics and transport in growth cone motility and axon guidance. Neuron 40:209-227. 
Dent EW, Kalil K (2001) Axon branching requires interactions between dynamic microtubules and actin filaments. J Neurosci 21:9757-9769.

Edelmann W, Zervas M, Costello P, Roback L, Fischer I, Hammarback JA, Cowan N, Davies P, Wainer B, Kucherlapati R (1996) Neuronal abnormalities in microtubule-associated protein 1B mutant mice. Proc Natl Acad Sci USA 93:1270-1275.

Fischer I, Romano-Clarke G (1990) Changes in microtubule-associated protein MAP1B phosphorylation during rat brain development. J Neurochem 55:328-333.

Gallo G, Letourneau PC (1998) Localized sources of neurotrophins initiate axon collateral sprouting. J Neurosci 18:5403-5414.

Gallo G, Letourneau PC (1999) Different contributions of microtubule dynamics and transport to the growth of axons and collateral sprouts. J Neurosci 19:3860-3873.

Gonzalez-Billault C, Avila J, Caceres A (2001) Evidence for the role of MAP1B in axon formation. Mol Biol Cell 12:2087-2098.

Gonzalez-Billault C, Owen R, Gordon-Weeks PR, Avila J, 1 (2002) Microtubule-associated protein $1 \mathrm{~B}$ is involved in the initial stages of axonogenesis in peripheral nervous system cultured neurons. Brain Res 943:56-67.

Goold RG, Owen R, Gordon-Weeks PR (1999) Glycogen synthase kinase 3beta phosphorylation of microtubule-associated protein $1 \mathrm{~B}$ regulates the stability of microtubules in growth cones. J Cell Sci 112:3373-3384.

Gordon-Weeks PR (1991a) Control of microtubule assembly in growth cones. J Cell Sci Suppl 15:45-49.

Gordon-Weeks PR (1991b) Growth cones: the mechanism of neurite advance. BioEssays 13:235-239.

Gordon-Weeks PR (1991c) Evidence for microtubule capture by filopodial actin filaments in growth cones. NeuroReport 2:573-576.

Gordon-Weeks PR (1993) Organization of microtubules in axonal growth cones: a role for microtubule-associated protein MAP 1B. J Neurocytol 22:717-725.

Gordon-Weeks PR, Fischer I (2000) MAP1B expression and microtubule stability in growing and regenerating axons. Microsc Res Tech 48:63-74.

Gundersen GG, Kalnoski MH, Bulinski JC (1984) Distinct populations of microtubules: tyrosinated and nontyrosinated alpha tubulin are distributed differently in vivo. Cell 38:779-789.

Hirokawa N (1994) Microtubule organization and dynamics dependent on microtubule-associated proteins. Curr Opin Cell Biol 6:74-81.

Homma N, Takei Y, Tanaka Y, Nakata T, Terada S, Kikkawa M, Noda Y, Hirokawa N (2003) Kinesin superfamily protein 2A (KIF2A) functions in suppression of collateral branch extension. Cell 114:229-239.

Kreis TE (1987) Microtubules containing detyrosinated tubulin are less dynamic. EMBO J 6:2597-2606.

Lin CH, Forscher P (1993) Cytoskeletal remodeling during growth conetarget interactions. J Cell Biol 121:1369-1383.

Lindsay RM (1988) Nerve growth factors (NGF, BDNF) enhance axonal regeneration but are not required for survival of adult sensory neurons. J Neurosci 8:2394-2405.

Ma D, Nothias F, Boyne LJ, Fischer I (1997) Differential regulation of microtubule-associated protein $1 \mathrm{~B}$ (MAP1B) in rat CNS and PNS during development. J Neurosci Res 49:319-332.

Ma D, Connors T, Nothias F, Fischer I (2000) Regulation of the expression and phosphorylation of microtubule-associated protein $1 \mathrm{~B}$ during regeneration of adult dorsal root ganglion neurons. Neuroscience 99:157-170.

Mack TG, Koester MP, Pollerberg GE (2000) The microtubule-associated protein MAP1B is involved in local stabilization of turning growth cones. Mol Cell Neurosci 15:51-65.

Matsuyama A, Shimazu T, Sumida Y, Saito A, Yoshimatsu Y, SeigneurinBerny D, Osada H, Komatsu Y, Nishino N, Khochbin S, Horinouchi S, Yoshida M (2002) In vivo destabilization of dynamic microtubules by HDAC6-mediated deacetylation. EMBO J 21:6820-6831.

Matus A (1990) Microtubule-associated proteins and the determination of neuronal form. J Physiol (Paris) 84:134-137.

Meixner A, Haverkamp S, Wassle H, Fuhrer S, Thalhammer J, Kropf N, Bittner RE, Lassmann H, Wiche G, Propst F (2000) MAP1B is required for axon guidance and Is involved in the development of the central and peripheral nervous system. J Cell Biol 151:1169-1178.

Noiges R, Eichinger R, Kutschera W, Fischer I, Nemeth Z, Wiche G, Propst F (2002) Microtubule-associated protein 1A (MAP1A) and MAP1B: light chains determine distinct functional properties. J Neurosci 22:2106-2114.
Nothias F, Fischer I, Murray M, Mirman S, Vincent JD (1996) Expression of a phosphorylated isoform of MAP1B is maintained in adult central nervous system areas that retain capacity for structural plasticity. J Comp Neurol 368:317-334.

Nothias F, Vernier P, von Boxberg Y, Mirman S, Vincent JD (1997) Modulation of NCAM polysialylation is associated with morphofunctional modifications in the hypothalamo-neurohypophysial system during lactation. Eur J Neurosci 9:1553-1565.

Owen R, Gordon-Weeks PR (2003) Inhibition of glycogen synthase kinase 3 beta in sensory neurons in culture alters filopodia dynamics and microtubule distribution in growth cones. Mol Cell Neurosci 23:626-637.

Pedrotti B, Islam K (1996) Dephosphorylated but not phosphorylated microtubule associated protein MAP1B binds to microfilaments. FEBS Lett 388:131-133.

Piperno G, LeDizet M, Chang XJ (1987) Microtubules containing acetylated alpha-tubulin in mammalian cells in culture. J Cell Biol 104:289-302.

Robson SJ, Burgoyne RD (1989) Differential localisation of tyrosinated, detyrosinated, and acetylated alpha-tubulins in neurites and growth cones of dorsal root ganglion neurons. Cell Motil Cytoskeleton 12:273-282.

Schulze E, Asai DJ, Bulinski JC, Kirschner M (1987) Posttranslational modification and microtubule stability. J Cell Biol 105:2167-2177.

Soares S, Fischer I, Ravaille-Veron M, Vincent JD, Nothias F (1998) Induction of MAP1B phosphorylation in target-deprived afferent fibers after kainic acid lesion in the adult rat. J Comp Neurol 396:193-210.

Soares S, von Boxberg Y, Lombard MC, Ravaille-Veron M, Fischer I, Eyer J, Nothias F (2002) Phosphorylated MAP1B is induced in central sprouting of primary afferents in response to peripheral injury but not in response to rhizotomy. Eur J Neurosci 16:593-606.

Szebenyi G, Callaway JL, Dent EW, Kalil K (1998) Interstitial branches develop from active regions of the axon demarcated by the primary growth cone during pausing behaviors. J Neurosci 18:7930-7940.

Szebenyi G, Dent EW, Callaway JL, Seys C, Lueth H, Kalil K (2001) Fibroblast growth factor-2 promotes axon branching of cortical neurons by influencing morphology and behavior of the primary growth cone. J Neurosci 21:3932-3941.

Takei Y, Kondo S, Harada A, Inomata S, Noda T, Hirokawa N (1997) Delayed development of nervous system in mice homozygous for disrupted microtubule-associated protein 1B (MAP1B) gene. J Cell Biol 137:1615-1626.

Takemura R, Okabe S, Umeyama T, Kanai Y, Cowan NJ, Hirokawa N (1992) Increased microtubule stability and alpha tubulin acetylation in cells transfected with microtubule-associated proteins MAP1B, MAP2 or tau. J Cell Sci 103:953-964.

Tanaka E, Sabry J (1995) Making the connection: cytoskeletal rearrangements during growth cone guidance. Cell 83:171-176.

Tanaka E, Ho T, Kirschner MW (1995) The role of microtubule dynamics in growth cone motility and axonal growth. J Cell Biol 128:139-155.

Tanaka EM, Kirschner MW (1991) Microtubule behavior in the growth cones of living neurons during axon elongation. J Cell Biol 115:345-363.

Thompson WC, Asai DJ, Carney DH (1984) Heterogeneity among microtubules of the cytoplasmic microtubule complex detected by a monoclonal antibody to alpha tubulin. J Cell Biol 98:1017-1025.

Tögel M, Wiche G, Propst F (1998) Novel features of the light chain of microtubule-associated protein MAP1B: microtubule stabilization, self interaction, actin filament binding, and regulation by the heavy chain. J Cell Biol 143:695-707.

Tonge DA, Aaronson OS, Golding JP, Jaggers D (1996) Cellular migration and axonal outgrowth from adult mammalian peripheral nerves in vitro. J Neurobiol 29:151-164.

Tonge DA, Golding JP, Edbladh M, Kroon M, Ekstrom PE, Edstrom A (1997) Effects of extracellular matrix components on axonal outgrowth from peripheral nerves of adult animals in vitro. Exp Neurol 146:81-90.

Tucker RP, Matus AI (1988) Microtubule-associated proteins characteristic of embryonic brain are found in the adult mammalian retina. Dev Biol 130:423-434

Webster DR, Borisy GG (1989) Microtubules are acetylated in domains that turn over slowly. J Cell Sci 92:57-65.

Webster DR, Gundersen GG, Bulinski JC, Borisy GG (1987) Differential turnover of tyrosinated and detyrosinated microtubules. Proc Natl Acad Sci USA 84:9040-9044.

Webster DR, Wehland J, Weber K, Borisy GG (1990) Detyrosination of alpha tubulin does not stabilize microtubules in vivo. J Cell Biol 111:113-122. 